\title{
Effect of glucose syrup solid, sucrose, hydrogenated palm oil and soy-lecithin on sensory acceptability of durian leather
}

\begin{abstract}
A two-stage optimization of ingredients in durian leather formulations was conducted using Response Surface Methodology (RSM). In stage 1, the independent variables were glucose syrup solids (GSS) and sucrose, and in stage 2, the variables were hydrogenated palm oil (HPO) and soy-lecithin (SL). Based on the responses to sensory acceptability attributes including taste, aroma, texture, appearance and overall acceptability, the most acceptable formulation was a combination of $10 \%$ GSS, 5\% sucrose, $2.67 \%$ HPO and $0.452 \%$ SL. Chemical analysis also showed that during processing nutrient composition was relatively unchanged.
\end{abstract}

Keyword: Durian leather; Sensory acceptability 